\title{
Prevendo os efeitos da mudança de produto em uma pequena empresa: eco-eficiência e produtividade global
}

\author{
Nilson Carvalho' \\ Recebido em: 08/11/2014. Aprovado em: 03/12/2014. Disponibilizado em: 26/12/2014
}

1. Nilson Carvalho, B. F. Giannetti, F. Agostinho and C. M.V.B. Almeida. São mestrandos no Programa de Pós - Graduação em Engenharia de Produção da Universidade Paulista. Rua Dr Bacelar 1212, Mirandópolis, 04026-002 - São Paulo, Brasil. nilsonmestrado@gmail.com.

\section{Resumo}

A ideia de avaliar produtos sob a perspectiva de cadeia de suprimentos para auxiliar na redução do impacto ambiental e socioeconômico associados à manufatura foi recentemente reconhecida. No entanto, não há estudos considerando os vários tipos de pequenas empresas que surgem na esfera de influência das grandes cadeias de abastecimento, a fim de preencher as lacunas ou servir clientes com necessidades especiais. Estas pequenas empresas que, em geral, não têm nenhuma influência sobre as decisões tomadas ao longo da cadeia de abastecimento, têm que adaptar seus processos de produção de acordo com as decisões tomadas pelas empresas líderes da cadeia. Este trabalho avalia a mudança de produto, com consequentes mudanças no uso de recursos ambientais e energia, de uma pequena empresa que opera no mercado brasileiro de reposição automotiva, usando síntese em emergia e confirma a ideia de que ações na cadeia de suprimentos principal podem ter efeitos benéficos. A simulação realizada para o período 2014-2025, dentro do plano de negócios da empresa, mostra que quanto mais cedo a troca de produtos for feita, maior será o lucro da empresa, maior a economia de recursos e energia e, portanto, maior sua eco-eficiência.

Palavras-chave: emergia; aftermarket automotivo, uso de recursos, eco-preço.

\begin{abstract}
The idea of evaluating products by the supply chain perspective in order to help the environmental impact reduce and socioeconomic associated to manufacturing has recently been recognized. However, there is no studies taking into consideration the various types of small companies that arise in the influence sphere of large supply chains in order to fill the gaps or serve customers with special needs. These small companies which, in general, have no influence on the decisions made along the supply chain. They have to adapt their production processes according to the decisions taken by the chain leading companies. This work evaluates the product changing with consequent changes by the use of environmental resources and energy of a small company operating in Brazilian market of automotive replacement,
\end{abstract}


using synthesis emerged and confirms the idea that actions in the chain of main supplies may have beneficial effects. The simulation performed for the period

2014-2025, within the company's business plan showed the earlier the exchange of products is made the greater a company profit, the greater tresources saving and energy, and, therefore the greater its eco-efficiency.

Keywords: emerging; automotive aftermarket, use of resources, eco-price. 


\section{Introdução}

As atividades de fabricação e manufatura contribuem significativamente para o uso de recursos. Com a expansão das atividades econômicas, as preocupações ambientais estão ganhando cada vez mais atenção e ações ambientais estão sendo desenvolvidas no sentido de prevenir os impactos causados pela manufatura e pelos processos de fabricação. Recentemente, se tem reconhecido a importância da adoção de estratégias holísticas por parte das autoridades e empresas, em que o uso de energia e recursos em toda a cadeia de valor dos produtos são examinados. Nesse contexto, são adotadas abordagens que avaliam toda a cadeia de suprimentos, com o objetivo de reduzir o impacto ambiental e socioeconômico associado ao fornecimento de produtos e serviços.

No Brasil, na esfera de influência de grandes cadeias de abastecimento, surgem também vários tipos de pequenas empresas, a fim de preencher as lacunas ou servir os clientes com necessidades especiais. Estas pequenas empresas, em geral, têm pouca ou nenhuma influência sobre as decisões tomadas pelas empresas líderes da cadeia. Entretanto, essas decisões acabam causando efeitos, positivos ou não, tanto no que diz respeito à receita dessas empresas de pequeno porte, como no impacto ambiental que elas podem causar individualmente.

Entre os métodos holísticos atualmente empregados, a síntese em emergia fornece uma avaliação complementar à da economia e, ao mesmo tempo, é aderente às leis fundamentais da termodinâmica, já que considera os limites biofísicos dos sistemas em estudo. Esse método de contabilidade ambiental, que considera a energia investida para a implantação e operação de sistemas ambientais e humanos, foi desenvolvido por Odum (1996). O método reconhece as relações entre a produção das matériasprimas pela natureza e seu consumo pelo homem, quantificando todo o investimento requerido em uma unidade física equivalente, que pode ser então convertida em valores monetários. A síntese em emergia tem sido usada com sucesso para avaliar vários processos de fabricação.

Cao e Feng (2007) analisaram a utilização de recursos e desempenho ambiental de um sistema de multi-produto, que foi dividido em duas categorias: sistemas de multi-produtos inseparáveis e sistemas de multi-produtos semi-independentes. Estes autores propuseram que, se o produto pertence a um sistema de multi-produto inseparável, sua emergia é igual ao de todo o sistema, mas se ele pertence a um sistema multi-produto semi-independente, as entradas devem ser alocadas. Também com base na síntese em emergia, Geng et al. (2010), destacam as relações internas entre os diferentes subsistemas de um parque industrial. Os resultados fornecem insights sobre o desempenho ambiental e a sustentabilidade do parque industrial de Dalian, localizado na Zona de Desenvolvimento Econômico na China, e mostram o potencial da síntese em emergia para elaboração de políticas ambientais, destacando as vantagens e limitações da análise para futuras pesquisas. $\mathrm{O}$ impacto das emissões de resíduos sobre o meio ambiente foi avaliado por $\mathrm{Mu}$ et al. (2011), que propõem um fator de amplificação para melhorar a informação fornecida pelos indicadores em emergia. Esses indicadores em emergia modificados foram utilizados para avaliar a incorporação da gestão de resíduos em um processo de produção de polietileno comercial.

Em um estudo mais abrangente, Giannetti et al. (2008), relatam as experiências de uma média empresa de semi-jóias, localizada em São Paulo, Brasil, a fim de reduzir o desperdício e a poluição. As 
medidas tomadas e as ações de Produção mais Limpa que foram introduzidas, assim como a sua relação custo-eficácia e os benefícios adicionais realizados foram apresentados e discutidos por meio da utilização de indicadores de escala local e global. Os resultados de um programa de minimização de resíduos e de intervenções de Produção Mais Limpa foram avaliados com o uso de indicadores como a intensidade de materiais (Ecological Rucksac) e a síntese em emergia, e mostraram que pequenas mudanças dentro da empresa, reduziram os impactos relativos ao uso de recursos e energia. Além disso, comprovou-se que os benefícios para o ambiente são maiores do que aqueles considerados apenas nas vizinhanças da empresa.

Os impactos ambientais e à saúde humana foram considerados ao se avaliar as opções para reduzir ou eliminar o chumbo do ambiente de produção de soldas brandas e a sua substituição por ligas sem chumbo (ALMEIDA et al., 2013), com e sem a utilização de logística reversa para recuperação de resíduos. Com o uso da síntese em emergia em conjunto com a Avaliação de Ciclo de Vida e o indicador DALY (Disability Adjusted Life Years), mostrou-se que mais recursos são usados para produzir uma tonelada de solda sem chumbo do que para produzir uma tonelada da liga de estanho-chumbo. A avaliação de emissões ao ar durante a produção de solda mostra que os benefícios da liga isenta de chumbo estão limitados às etapas de fabricação e de montagem. A solda de estanho-chumbo foi apontada como a melhor opção em termos de eficiência no uso de recursos e no que diz respeito às emissões para a atmosfera, quando toda a cadeia de abastecimento é considerada.

O objetivo deste trabalho é avaliar e prever as mudanças no uso de recursos ambientais de uma pequena empresa, que opera no mercado de reposição automotiva brasileira, devido à troca de produto imposta pelas empresas líderes da cadeia de suprimentos. O efeito da mudança de produto é avaliado com relação à eficiência na utilização dos recursos, produtividade, carga ambiental e produtividade global.

\section{Descrição da empresa}

A JP JUNTAS iniciou suas atividades no mercado de reposição automotiva em 1992, e atua em uma cadeia de distribuição que exige grande diversidade de produtos e serviços para o atendimento de seus clientes. A especialidade da empresa é a fabricação de produtos de vedação automotiva, que envolvem mais de 1.500 itens. A empresa, que iniciou suas atividades fornecendo juntas para motores com carburador, teve que se adaptar à nova demanda por juntas para motores com injeção de combustível eletrônica. Hoje, a empresa fornece os dois tipos de juntas, com uma produção média de meio milhão de unidades por ano. A meta da empresa é consolidar sua presença na cadeia automotiva nos próximos anos, substituindo totalmente a produção de juntas de carburadores pelas juntas de injeção eletrônica até 2025 .

\section{Métodos}

Emergia é definida como a energia disponível anteriormente requerida, direta e indiretamente, para a obtenção de um produto ou serviço (ODUM, 1996). A avaliação em emergia converte todas as contribuições recebidas pelo sistema de produção (metais, energia, petróleo e moeda) em uma única base de medição (joules de energia solar, seJ).

A coleta de dados foi efetuada pela consulta a documentos formais de compra, no ano de 2013, de acordo com as seguintes etapas: 
(I) estudo do contexto em que está inserido o sistema,

(II) a definição do sistema em estudo, incluindo os seus limites para a investigação e descrição dos processos de fabricação,

(III) a execução do balanço de massa e construção de fluxos de energia (diagrama),

(IV) a construção das tabelas de emergia com os dados recolhidos, e

(V) a discussão para ações futuras.

Para cada tipo de junta, as quantidades de energia elétrica consumida, água, papel e papelão, óleo, lubrificante e trabalho, foram calculados. As quantidades de materiais usados na implantação do sistema foram contabilizadas considerando a sua vida útil. Os valores unitários em emergia (UEVs) utilizados neste texto são tomados principalmente da literatura e são relativos a 15,83 x 1024 seJ / ano base (ODUM et al., 2000).

O Emprice (ODUM, 1996) ou ecopreço (CAMPBELL e TILLEY, 2013) tem o objetivo de representar de forma mais precisa o valor dos recursos ambientais do que a abordagem alternativa de desejo de pagar (willigness to pay). O Emprice é o quociente entre a emergia e o produto interno bruto (PIB) da região em que o sistema está inserido. A emergia em relação ao dólar do Estado de São Paulo é de 1,7 x 1012 seJ / U\$ (DEMÉTRIO, 2007). A Emergia em relação ao dólar da empresa também é calculada, pelo quociente entre a emergia total e a receita da empresa. A produtividade global (PG), semelhante para a produtividade comum, é a razão entre as entradas e as saídas. Entretanto, como a PG inclui recursos, energia e serviços da biosfera incorporados nas UEVs, é, consequentemente, mais ampla e completa do que a produtividade normalmente calculada.

\section{Resultados}

A Figura 1 mostra o diagrama de energia da companhia.

Figura 1: Diagrama de energia da JP JUNTAS.

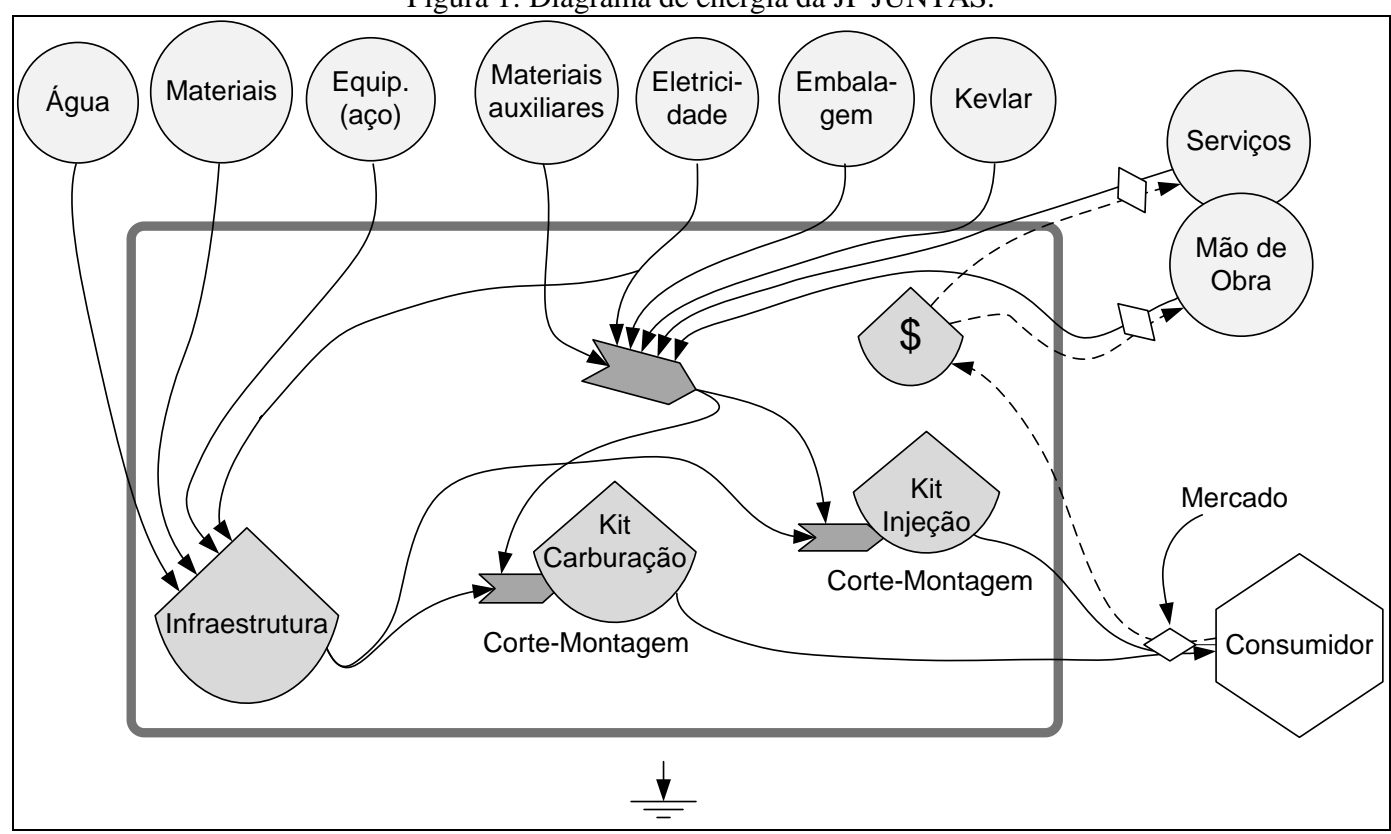

Fonte: produzida pelo autor em São Paulo, 2014 
A Tabela 1 mostra a contabilidade ambiental em emergia da empresa em 2013. Neste ano, quantidades iguais de ambos os tipos de juntas foram produzidos. No entanto, observa-se que a emergia investida na produção de kits de carburador foi duas vezes maior do que a utilizada para produzir a mesma quantidade de kits de injeção eletrônica. Os custos em emergia da empresa, representados pelo custo anual do edifício, instalações, escritórios e máquinas, são responsáveis por apenas $7 \%$ do custo anual total. A produção do kit de carburador corresponde a $67,4 \%$ do total de emergia e, como esperado, a maior contribuição para a emergia total está associada ao uso de mantas de Kevlar, seguida pela manta de celulose.

Tabela 1. Contabilidade ambiental em emergia da JP Juntas em 2013.

\begin{tabular}{|c|c|c|c|c|c|c|}
\hline & & Quant. & Unid. & UEVs/(seJ/unid.) & Emergia/(seJ/ano) & $\%$ \\
\hline & Instalações da empresa & & & & & \\
\hline 1 & Concreto & $2,62 \times 10^{7}$ & $\mathrm{~g}$ & $1,23 \times 10^{9}$ & $3,22 \times 10^{16}$ & 2,4 \\
\hline 2 & Aço & $7,85 \times 10^{5}$ & $\mathrm{~g}$ & $5,31 \times 10^{9}$ & $4,17 \times 10^{15}$ & 0,3 \\
\hline \multirow[t]{2}{*}{3} & Madeira & $5,32 \times 10^{5}$ & $\mathrm{~g}$ & $4,04 \times 10^{8}$ & $2,15 \times 10^{14}$ & $<0,1$ \\
\hline & Área administrativa & & & & & \\
\hline 4 & Plástico & $1,86 \times 10^{4}$ & $\mathrm{~g}$ & $3,80 \times 10^{8}$ & $7,07 \times 10^{12}$ & $<0,1$ \\
\hline 5 & Cobre & $4,54 \times 10^{3}$ & $\mathrm{~g}$ & $6,80 \times 10^{10}$ & $3,09 \times 10^{14}$ & $<0,1$ \\
\hline 6 & Aço & $1,64 \times 10^{6}$ & $\mathrm{~g}$ & $5,31 \times 10^{9}$ & $8,71 \times 10^{15}$ & 0,6 \\
\hline \multirow[t]{2}{*}{7} & Vidro & $2,00 \times 10^{3}$ & $\mathrm{~g}$ & $8,40 \times 10^{8}$ & $1,68 \times 10^{12}$ & $<0,1$ \\
\hline & Planta de produção & & & & & \\
\hline 8 & Plástico & $3,76 \times 10^{5}$ & $\mathrm{~g}$ & $3,80 \times 10^{8}$ & $1,43 \times 10^{14}$ & $<0,1$ \\
\hline \multirow[t]{3}{*}{9} & Aço 1 & $8,92 \times 10^{6}$ & $\mathrm{~g}$ & $5,31 \times 10^{9}$ & $4,74 \times 10^{16}$ & 3,5 \\
\hline & Sub-total 1 & & & & $9,30 \times 10^{16}$ & 7 \\
\hline & Kit de carburação & & & & & \\
\hline 10 & Água & $8,48 \times 10^{7}$ & $\mathrm{~g}$ & $6,64 \times 10^{5}$ & $5,63 \times 10^{13}$ & $<0,1$ \\
\hline 11 & Eletricidade & $2,30 \times 10^{11}$ & $\mathrm{~J}$ & $1,28 \times 10^{5}$ & $2,94 \times 10^{16}$ & 2,2 \\
\hline 12 & Mão de Obra & $6,20 \times 10^{9}$ & $\mathrm{~J}$ & $1,15 \times 10^{7}$ & $7,13 \times 10^{16}$ & 5,2 \\
\hline 13 & Lubrificante & $8,65 \times 10^{9}$ & $\mathrm{~J}$ & $5,91 \times 10^{4}$ & $5,11 \times 10^{14}$ & $<0,1$ \\
\hline 14 & Borracha (o'rings) & $9,60 \times 10^{4}$ & $\mathrm{~g}$ & $2,10 \times 10^{4}$ & $2,02 \times 10^{9}$ & $<0,1$ \\
\hline 15 & Cobre (diafragmas e molas) & $8,64 \times 10^{5}$ & $\mathrm{~g}$ & $6,80 \times 10^{10}$ & $5,88 \times 10^{16}$ & 4,3 \\
\hline 16 & Zinco (diafragmas e molas) & $1,30 \times 10^{6}$ & $\mathrm{~g}$ & $6,80 \times 10^{10}$ & $8,81 \times 10^{16}$ & 6,5 \\
\hline 17 & Mangueiras de borracha & $1,68 \times 10^{6}$ & $\mathrm{~g}$ & $2,10 \times 10^{4}$ & $3,53 \times 10^{10}$ & $<0,1$ \\
\hline 18 & Manta de celulose & $2,78 \times 10^{7}$ & $\mathrm{~g}$ & $3,90 \times 10^{9}$ & $1,09 \times 10^{17}$ & 8,0 \\
\hline 19 & Kevlar vermelho & $1,34 \times 10^{6}$ & $\mathrm{~g}$ & $1,25 \times 10^{10}$ & $1,68 \times 10^{16}$ & 1,2 \\
\hline 20 & Kevlar verde & $3,76 \times 10^{7}$ & $\mathrm{~g}$ & $1,25 \times 10^{10}$ & $4,70 \times 10^{17}$ & 34,6 \\
\hline 21 & Papel & $4,80 \times 10^{5}$ & $\mathrm{~g}$ & $3,90 \times 10^{9}$ & $1,87 \times 10^{15}$ & 0,1 \\
\hline 22 & Papelão & $1,58 \times 10^{6}$ & $\mathrm{~g}$ & $3,90 \times 10^{9}$ & $6,18 \times 10^{15}$ & 0,5 \\
\hline 23 & Etiquetas & $2,40 \times 10^{5}$ & $\mathrm{~g}$ & $3,90 \times 10^{9}$ & $9,36 \times 10^{14}$ & 0,1 \\
\hline 24 & Blister & $5,04 \times 10^{3}$ & $\mathrm{~g}$ & $3,80 \times 10^{8}$ & $1,92 \times 10^{12}$ & $<0,1$ \\
\hline \multirow[t]{3}{*}{25} & Sacos plásticos & $2,38 \times 10^{5}$ & $\mathrm{~g}$ & $3,80 \times 10^{8}$ & $9,03 \times 10^{13}$ & $<0,1$ \\
\hline & Sub-total 2 & & & & $9,16 \times 10^{17}$ & 67,4 \\
\hline & Kit de injeção eletrônica & & & & & \\
\hline 26 & Água & $8,48 \times 10^{7}$ & $\mathrm{~g}$ & $6,64 \times 10^{5}$ & $5,63 \times 10^{13}$ & $<0,1$ \\
\hline 27 & Eletricidade & $9,84 \times 10^{10}$ & $\mathrm{~J}$ & $1,28 \times 10^{5}$ & $1,26 \times 10^{16}$ & 0,9 \\
\hline 28 & Mão de Obra & $6,20 \times 10^{9}$ & $\mathrm{~J}$ & $1,15 \times 10^{7}$ & $7,13 \times 10^{16}$ & 5,2 \\
\hline 29 & Lubrificante & $8,65 \times 10^{9}$ & $\mathrm{~J}$ & $5,91 \times 10^{4}$ & $5,11 \times 10^{14}$ & $<0,1$ \\
\hline 30 & Borracha (o'rings) & $1,34 \times 10^{6}$ & $\mathrm{~g}$ & $2,10 \times 10^{4}$ & $2,82 \times 10^{10}$ & $<0,1$ \\
\hline
\end{tabular}

CARVALHO, N.: Prevendo os efeitos da mudança de produto em uma pequena empresa: 


\begin{tabular}{|c|c|c|c|c|c|c|}
\hline 31 & Kevlar verde & $2,42 \times 10^{7}$ & $\mathrm{~g}$ & $1,25 \times 10^{10}$ & $3,02 \times 10^{17}$ & 22,3 \\
\hline 32 & Papel & $4,80 \times 10^{5}$ & $\mathrm{~g}$ & $3,90 \times 10^{9}$ & $1,87 \times 10^{15}$ & 0,1 \\
\hline 33 & Papelão & $1,58 \times 10^{6}$ & $\mathrm{~g}$ & $3,90 \times 10^{9}$ & $6,18 \times 10^{15}$ & 0,5 \\
\hline 34 & Etiquetas & $2,40 \times 10^{5}$ & $\mathrm{~g}$ & $3,90 \times 10^{9}$ & $9,36 \times 10^{14}$ & 0,1 \\
\hline 35 & Blister & $9,60 \times 10^{4}$ & $\mathrm{~g}$ & $3,80 \times 10^{8}$ & $3,65 \times 10^{13}$ & $<0,1$ \\
\hline 36 & Sacos plásticos & $1,80 \times 10^{5}$ & $\mathrm{~g}$ & $3,80 \times 10^{8}$ & $6,84 \times 10^{13}$ & $<0,1$ \\
\hline & Sub-total 3 & & & & $4,43 \times 10^{17}$ & 32,6 \\
\hline & EmergiaTotal & & & & $1,45 \times 10^{18}$ & \\
\hline
\end{tabular}

Fonte: produzida pelo autor em São Paulo, 2014

A emergia total da empresa em 2013 considera todos os recursos e energia necessários para a produção de 480 mil kits de juntas para motores com carburador e injeção eletrônica (Tab. 1). As UEVs, que traduzem o uso de energia e recursos em termos de emergia por unidade de energia do produto, mostram que se emprega aproximadamente o dobro de emergia para produzir um kit de carburador (Tab.2), o que é desfavorável, tanto para o meio ambiente como para a empresa. Como o kit de carburador é vendido por U\$2,00 enquanto o kit de injeção eletrônica por U\$3,50, o valor da EMR dos kits também mostra que menos emergia é utilizada para gerar um dólar na produção de kits de injeção eletrônica.

Tabela 2. Resumo da contabilidade ambiental em emergia da JP Juntas em 2013.

\begin{tabular}{|l|l|l|l|}
\hline & Carburador & Injeção eletrônica & Total \\
\hline Total emergia 2013 (seJ/ano) & $9.63 \times 10^{17}$ & $4.89 \times 10^{17}$ & $1.45 \times 10^{18}$ \\
\hline UEV (seJ/kit) & $4.01 \times 10^{12}$ & $2.04 \times 10^{12}$ & $2,83 \times 10^{12}$ \\
\hline Emprice* (Em\$/ano) & 566,198 & 287,797 & 799,235 \\
\hline EMR** (seJ/U\$) & $2.01 \times 10^{12}$ & $5.82 \times 10^{11}$ & $1.10 \times 10^{12}$ \\
\hline Receitas (U\$/ano) & 480,000 & 840,000 & $1,320,000$ \\
\hline
\end{tabular}

Fonte: produzida pelo autor em São Paulo, 2014

* O Emprice é o quociente de emergia de dólares (1,7 x 1012 seJ / U\$, (DEMÉTRIO, 2007) da área onde o sistema está inserido.

** A emergia a relação dólar da empresa também é calculada, pelo quociente entre a emergia total e a receita da empresa.

A empresa planejou reduzir gradualmente a produção de kits de carburador até 2025. Desta forma, é possível, com o emprego da síntese em emergia, avaliar o impacto desta decisão gerencial na contabilidade ambiental da empresa nos próximos anos. A simulação levou em conta alguns fatores:
- a redução do número de kits de carburação será compensada pela produção de kits de injeção eletrônica;

- haverá a redução do uso de energia elétrica devido ao número reduzido de operações de corte na produção de kits de injeção eletrônica. A quantidade de energia elétrica a ser empregada foi calculada e contabilizada na proporção do número e tipo de kits produzidos;

- haverá redução da força de trabalho em 2020, que foi estimada de acordo com o número e tipo dos kits produzidos;

- o preço dos kits foi mantido constante. 
A Tabela 3 mostra os resultados da contabilidade ambiental da empresa até 2025. Fica claro que, com o aumento da percentagem de kits de injeção eletrônica, a emergia total da empresa diminui juntamente com a utilização de energia e materiais para produzir uma unidade de produto (UEVs). O Emprice, que traduz os custos em emergia em moeda, também diminuirá em $35 \%$, enquanto que a receita da empresa terá aumento de $24 \%$.

Tabela 3. Resumo da contabilidade ambiental em emergia da JP Juntas, no período de 2014-2025.

\begin{tabular}{|c|c|c|c|c|c|c|c|c|c|c|c|c|}
\hline & 2014 & 2015 & 2016 & 2017 & 2018 & 2019 & 2020 & 2021 & 2022 & 2023 & 2024 & 2025 \\
\hline \% Emergia kits de injeção & 37 & 41 & 45 & 50 & 54 & 60 & 64 & 70 & 77 & 84 & 92 & 100 \\
\hline Emergia total x $10^{18}(\mathrm{seJ} / \mathrm{ano})$ & 1,32 & 1,28 & 1,24 & 1,20 & 1,16 & 1,12 & 1,05 & 1,01 & 0,97 & 0,93 & 0,89 & 0,85 \\
\hline UEV x $10^{12}$ (seJ/kit) & 2,74 & 2,66 & 2,58 & 2,50 & 2,42 & 2,34 & 2,19 & 2,11 & 2,02 & 1,94 & 1,86 & 1,77 \\
\hline EmPrice x $10^{6}$ (Em\$/ano) & 0,8 & 0,8 & 0,7 & 0,7 & 0,7 & 0,7 & 0,6 & 0,6 & 0,6 & 0,5 & 0,5 & 0,5 \\
\hline Receita x $10^{6}$ (U\$/ano) & 1,35 & 1,38 & 1,41 & 1,44 & 1,47 & 1,5 & 1,53 & 1,56 & 1,59 & 1,62 & 1,65 & 1,68 \\
\hline
\end{tabular}

Fonte: produzida pelo autor em São Paulo, 2014

Também se observa que a mudança de produto, apesar de induzida pelas grandes empresas na cadeia de abastecimento, pode trazer benefícios adicionais para a JP JUNTAS. A Figura 2 mostra que a emergia incorporada em produtos pode diminuir em $31 \%$ até 2025 . Esta economia de emergia, isto é, a emergia que não será mais investida na produção dos kits de carburação, estará disponível para outros usos na biosfera. Prevê-se um decréscimo de $17 \%$ nos custos em emergia para a manutenção, o uso de materiais auxiliares e de mão de obra, e espera-se que o uso de materiais de embalagem possa diminuir significativamente em mais de $80 \%$, enquanto a receita da empresa aumenta.

Figura 2. Previsão para o uso de emergia em produtos, manutenção, materiais auxiliares, mão de obra e embalagem entre 2014 e 2025.

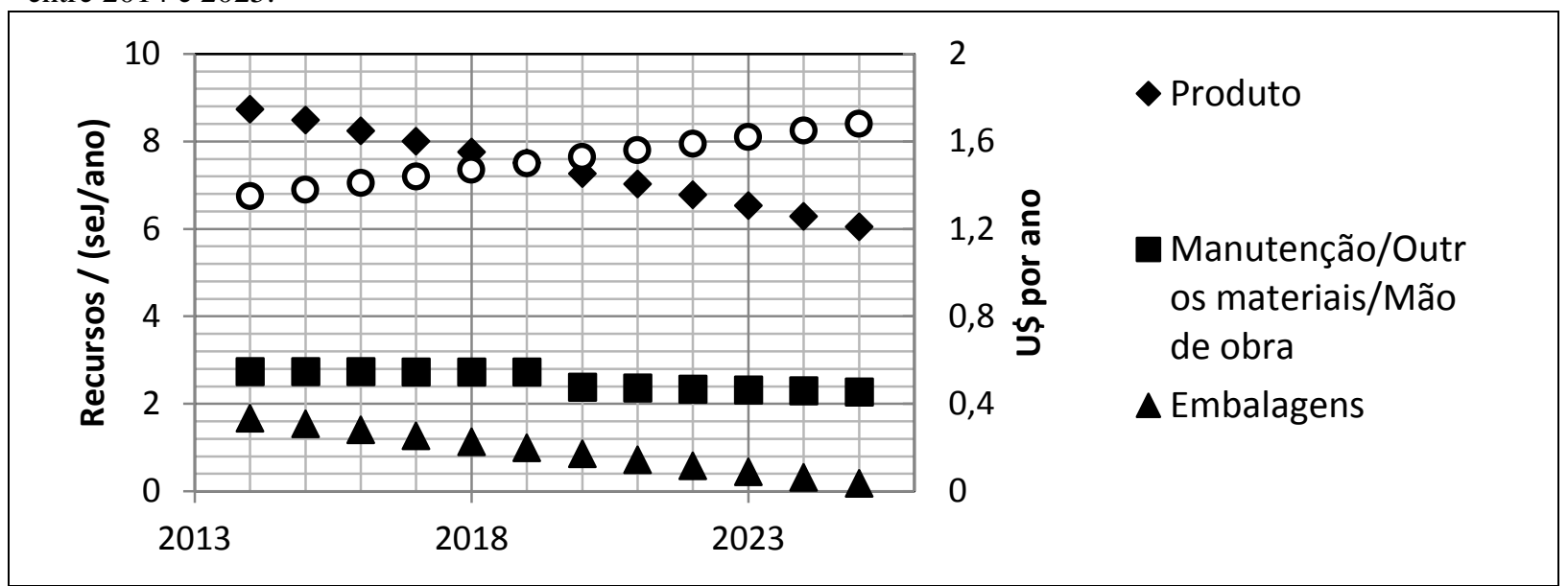

Fonte: desenvolvida pelo autor em São Paulo, 2014

No que diz respeito aos custos de emergia convertidos em valores monetários, os resultados mostrados na Figura 3 deixam claro que a mudança de produto irá beneficiar não só a empresa, reduzindo os custos ambientais em 35\%, mas também que a 
produtividade global vai aumentar em 55\%, devido ao menor uso de matéria prima, auxiliares, embalagem e energia.

Figura 3. Custo/Benefício e Produtividade global da JP JUNTAS no período de 2014 a 2025.

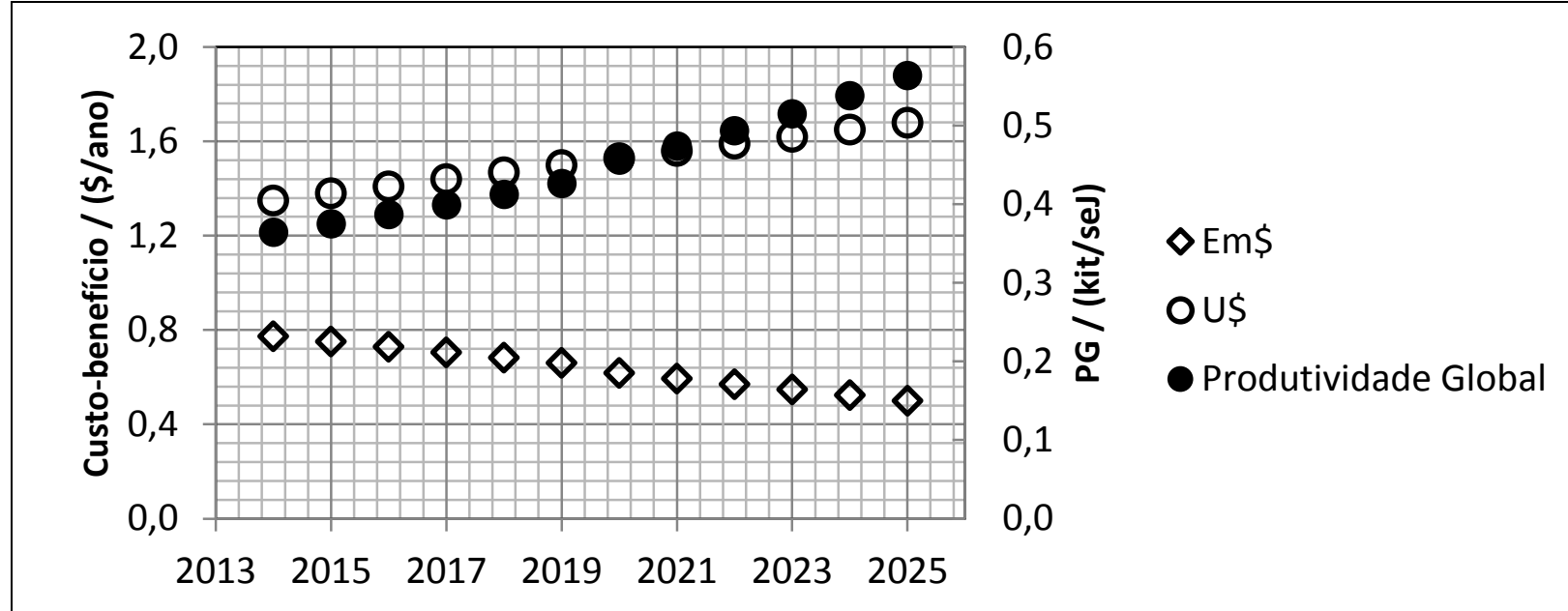

Fonte: produzida pelo autor em São Paulo, 2014

Os ganhos devido ao aumento da produtividade global não se restringem aos empresários e gestores do pequeno negócio, mas se estendem ao meio ambiente, uma vez que parte dos recursos e energia poupados pode ser usada para outros fins (Fig. 4). A relação Emergia/Receita, que diminui em $48 \%$, mostra que a mudança de produto torna a empresa mais eco-eficiente, já que lucra mais com menor uso de recursos e energia.

Figura 4. Previsão para a relação Emergia/Receita (EMR*) e para a Produtividade global da JP JUNTAS de 2014 a 2025.

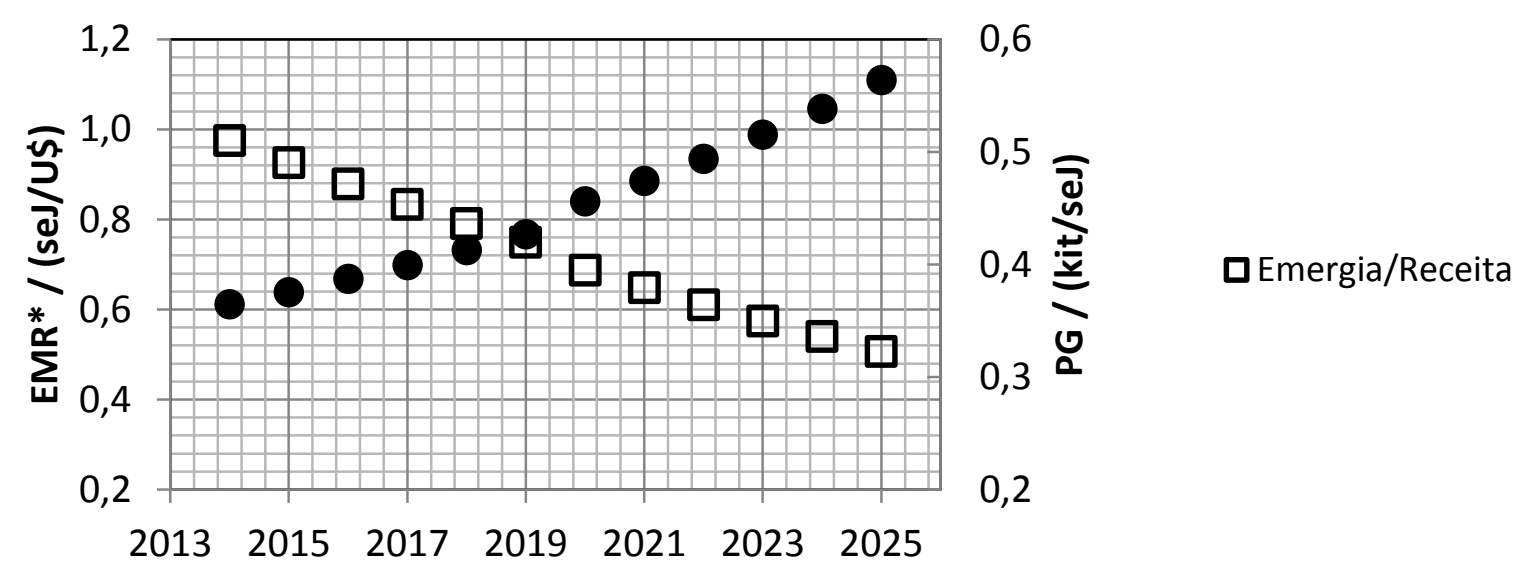

Fonte: produzida pelo autor em São Paulo, 2014 


\section{Considerações finais}

A avaliação de uma pequena empresa no mercado de reposição automotiva brasileira confirma a ideia de ações ao longo da cadeia de suprimentos podem contribuir para reduzir o impacto ambiental e a redução da carga associada à fabricação de produtos dentro e fora da cadeia de valor. Todos os resultados mostram que a mudança tecnológica, que ocorreu em outras partes da cadeia de abastecimento, tem efeitos benéficos sobre essa pequena empresa que existe e sobrevive em torno da cadeia principal sem, porém, participar dela diretamente.

Os efeitos no âmbito social, que parecem negativos devido ao corte de mão de obra previsto para 2020, poderão ser compensados se forem reavaliados à luz da aplicação de um programa de treinamento de pessoal, o que pode aumentar a possibilidade desses trabalhadores conseguirem melhores empregos ou exercerem funções de maior responsabilidade na própria empresa. Os resultados da simulação para o período de 2014 a 2025, dentro do plano de negócios da empresa, mostram que quanto mais cedo ocorrer a troca de produtos, maior será a ecoeficiência da empresa.

\section{Bibliografia}

ALMEIDA, C.M.V.B., MADUREIRA, M.A., BONILLA, S.H., GIANNETTI, B.F., (2013). Assessing the replacement of lead in solders: effects on resource use and human health. Journal of Cleaner Production, Volume 47, 457-464.

CAMPBELL, E.T., TILLEY, D.R., (2014). The eco-price: How environmental emergy equates to currency. (2014). Ecosystem Services, Volume 7, 128-140.

CAO, K., FENG, X., (2007). The Emergy Analysis of Multi-Product Systems Trans IChemE, Part B, Process Safety and Environmental Protection, 85 (B5): 494-500. DEMÉTRIO, J.F.C. (2011). Emergy Environmental Sustainability Assessment of Brazil, Ph. D.dissertation. Universidade Paulista, São Paulo, Brazil (in Portuguese). 〈http://www3.unip.br/ensino/pos_graduac ao/strictosensu/lab_producao_meio ambiente/realizações academicas.aspx\#ra4, último acesso em Setembro (2013).

GENG, Y., ZHANG, P., ULGIATI, S., SARKIS, J., (2010). Emergy analysis of an industrial park: The case of Dalian, China Science of the Total Environment 408, 52735283.

GIANNETTI， B.F., BONILLA， S.H., SILVA, I.R., ALMEIDA, C.M.V.B., (2008). Cleaner production practices in a medium size gold-plated jewelry company in Brazil: when little changes make the difference Journal of Cleaner Production 16,1106 e 1117.

MU, H., FENG, X., CHU, KH., (2011). Improved emergy indices for the evaluation of industrial systems incorporating waste management Ecological Engineering 37, $335-342$. 
ODUM, H.T., (1996), Environmental Accounting: Emergy and Environmental Decision Making. John Wiley \& Sons, New York p. 370.
ODUM, H.T.,ODUM, E.P., (2000).The energetic basis for valuing eco system services. 